\title{
Model and Operation of Electricity-Heat-Hydrogen Energy Station
}

\author{
Gang Chen ${ }^{1, ~ a ~}$, Lijie Ding ${ }^{1, b}$, Xiaohua Wang ${ }^{2, \mathrm{c}}$ and Huabo Shi ${ }^{1, \mathrm{~d}}$ \\ ${ }^{1}$ State Grid Sichuan Electric Power Research Institute, Chengdu 610072, China \\ ${ }^{2}$ Energy Internet Research Institute of Tsinghua University, Chengdu 610072, China \\ agangchen08@gmail.com, 'ding_lijie@163.com, cwangxiaohua@tsinghua-eiri.org, \\ shbo87@163.com
}

Keywords: Multi-energy microgrid; hydrogen; electrolysis cell; thermoelectric coupling.

Abstract. Hydrogen is expected to replace part of the fossil fuels in the future. Distributed wind and solar power is possible to produce hydrogen as an important primary energy source in order to enhance flexibility in energy distribution. The object of study is electricity-heat-hydrogen energy station composed of distributed power, electrolytic hydrogen, fuel cells and gas boiler. The research is focused on the models of hydrogen production and consumption to get the relationship among electricity/hydrogen/heat energy. A form of circuit diagram is used in this study. In addition, this paper also analyzes the station's economical running simulation model based on minimizing overall operating costs. The results prove that this system is actually capable of improving the overall efficiency of energy distribution.

\section{Introduction}

As a fuel with high energy density, extensive generation sources and clean combustion reaction, hydrogen is expected to become an important foundation of our transportation energy and replace part of the fossil fuels in the future. Since distributed generations like wind and solar are close to the hydrogen consumption centers, they can be integrated into hydrogen production by means of multi-energy microgrid so as to enhance energy flexibility and improve the utilization of distributed power [1, 2]. A typical example of such microgrids is shown in Fig. 1.

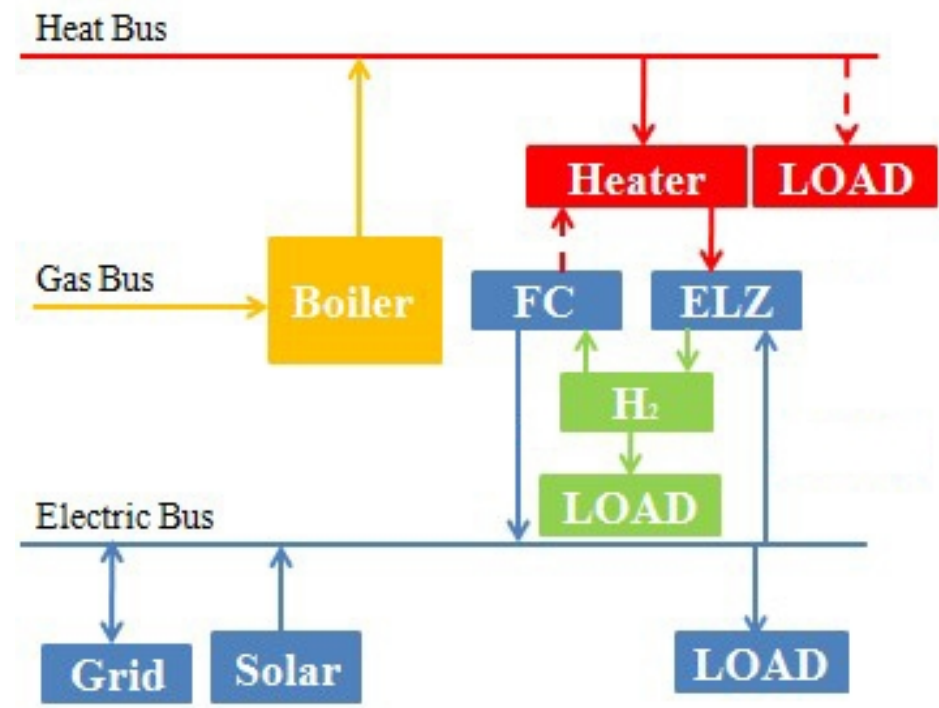

Fig. 1 Electricity-heat-hydrogen energy station (an example of multi-energy microgrid)

Some research about model and operation of hydrogen involved microgrids has been published. Reference [3] presents a domestic microgrids with hydrogen storage to take full advantage of renewable sources to supply electric vehicle. Photovoltaic, batteries, electrolyzer and fuel cell also take part in the forementioned system. In [4], an energy management strategy based on coordination of battery, electrolyzer and fuel cell is proposed to minimize the operation cost of multi-energy system. Pattern in different seasons is analyzed. 
This paper focus on the model and operation of electricity-heat-hydrogen energy station in Fig. 1. The coupling among distributed generation, electrolyzer, fuel cells and gas boiler will be analyzed, so that this microgrid can meet the electrical load, heat load as well as hydrogen demand with minimum cost.

\section{Modeling of Multi-energy Station}

Electrolyzer and Fuel Cell. Based on the published work about electrolyzer and fuel cell [5-7], the following electrochemical model can be extracted

$$
\begin{aligned}
& U_{e}=U_{r e v}\left(T_{e}\right)+U_{a c t}\left(T_{e}, i_{e}\right)+U_{\text {ohm }}\left(T_{e}, i_{e}\right) \\
& U_{f}=E_{\text {nernst }}\left(T_{f}\right)-U_{\text {conc }}\left(i_{f}\right)-U_{a c t}\left(i_{f}\right)-U_{\text {ohm }}\left(T_{f}, i_{f}\right) .
\end{aligned}
$$

The reversible voltage and irreversible voltages are dependent on temperature $T$ and current density $i$ of electrolyzer or fuel cell. The $U-T-i$ relationship shown in Eq. 1 and Eq. 2 can be illustrated with the surfaces in Fig. 2.
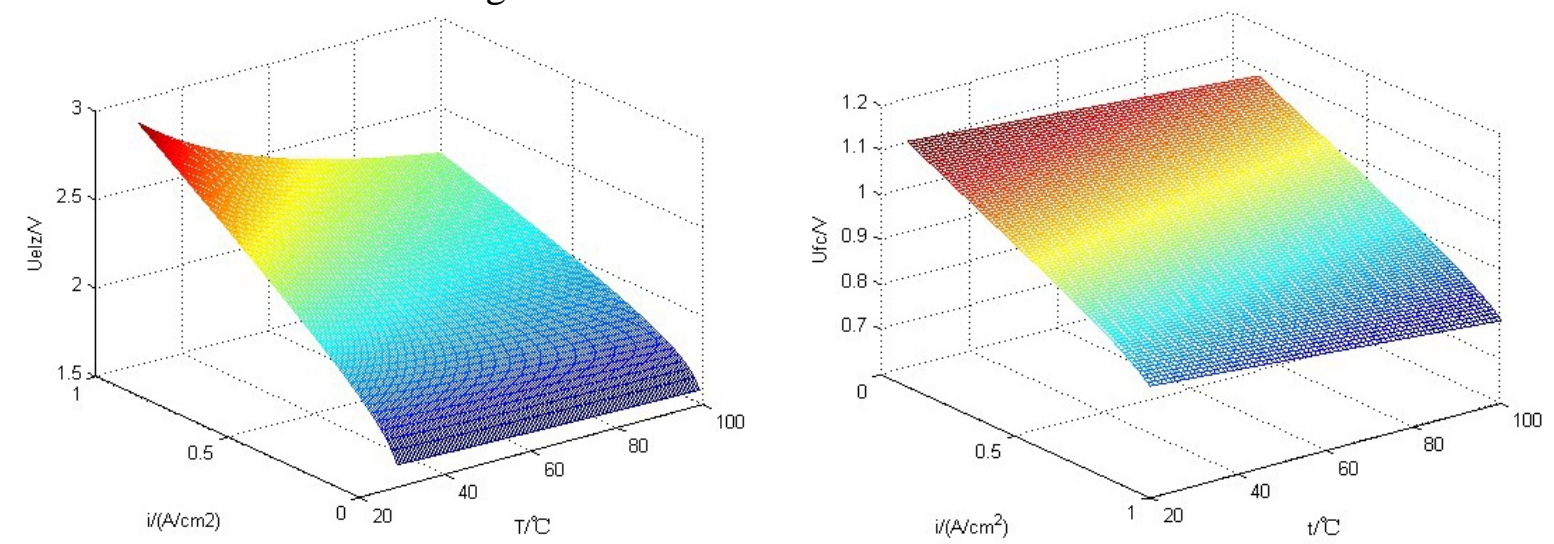

Fig. $2 U-T-i$ relationship of electrolyzer (left) and fuel cell (right)

Apperently the $U-T-i$ relationship above is non-linear in the whole acceptable temperature range. However, the surfaces are almost flat if the temperature is restricted to an interval of $\left[60^{\circ} \mathrm{C}, 80^{\circ} \mathrm{C}\right]$, which is the regular temperature range for electrolyzer or fuel cell operation. Actually for the case analyzed in this paper, the above $U-T-i$ relationship can be fitted by

$U_{e}\left(T_{e}, i_{e}\right)=1.975+0.5909 i_{e}-0.003038 T_{e}, T_{e} \in[60,80]$

$U_{f}\left(T_{f}, i_{f}\right)=1.195-0.3055 i_{f}-0.0008114 T_{f}, T_{f} \in[60,80]$.

Meanwhile, the hydrogen produced or consumed by electrolyzer or fuel cell can be determined by their operation current directly. The molar quantity of accumulated hydrogen during current sample period can be calculated by

$$
\Delta n_{H_{2}}=K_{e}^{H} i_{e}-K_{f}^{H} i_{f}
$$

where parameters $K_{e}^{H}, K_{f}^{H}$ can be derived from Faraday constant, sample time, cell number and reaction area. The input power of electrolyzer and output power of fuel cell can be expressed as

$$
P_{E L Z}=K_{e}^{P} U_{e} i_{e}
$$


$P_{F C}=K_{f}^{P} U_{f} i_{f}$

where parameters $K_{e}^{P}, K_{f}^{P}$ can be derived from cell number and reaction area.

Thermal Model. The heat transfer among components can be described as.

$$
\left\{\begin{array}{l}
C_{h} \frac{d T_{h}}{d t}=\frac{T_{a}-T_{h}}{R_{a h}}+\frac{T_{e}-T_{h}}{R_{e h}}+P_{H T} \\
C_{e} \frac{d T_{e}}{d t}=\frac{T_{a}-T_{e}}{R_{a e}}+\frac{T_{h}-T_{e}}{R_{e h}} \\
C_{f} \frac{d T_{f}}{d t}=\frac{T_{a}-T_{f}}{R_{a f}}+\frac{T_{h}-T_{f}}{R_{f h}}
\end{array}\right.
$$

where $T_{h}, T_{e}, T_{f}, T_{a}$ are temperatures of heater, electrolyzer, fuel cell and ambient environment. The important thermodynamic parameters for heat transfer [8] are $C_{i}$, i.e. thermal capacities of component $i$, and $R_{i j}$, i.e. thermal resistance between components $i$ and $j . P_{H T}$ is heat power injected into the heater from heat bus.

If we regard temperatures as virtual voltages of electrical circuit, a thermal circuit as shown in Fig. 3 can be proposed to illustrate the thermal coupling model of Eq. 8. The heat flows are compared to direct current in this circuit.

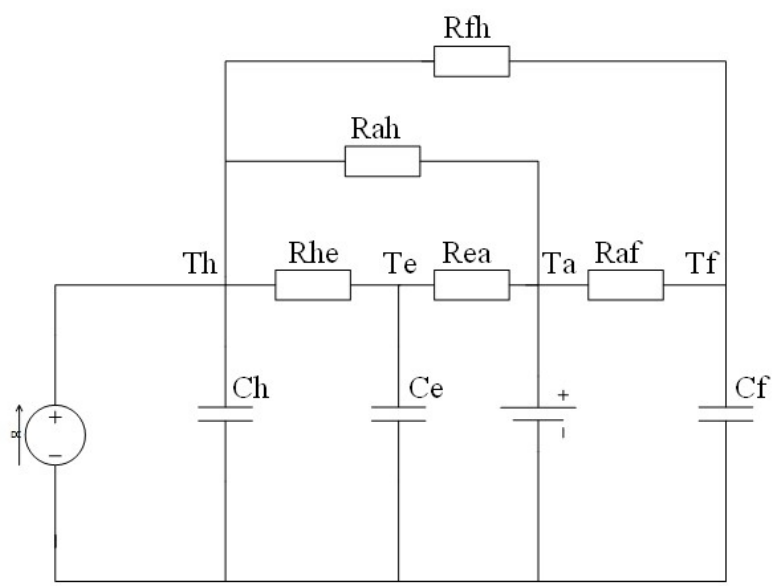

Fig. 3 Thermal circuit of electricity-heat-hydrogen energy station

Network Model. The following power balances (in terms of electricity and heat) should be fulfilled at all time

$$
\begin{gathered}
P_{P V}+P_{G R I D}+P_{F C}=P_{E L O A D}+P_{E L Z} \\
P_{G B}=P_{H L O A D}+P_{H T}
\end{gathered}
$$

where $P_{P V}, P_{G R I D}, P_{F C}, P_{G B}$ are power generation from photovoltaic, external grid, fuel cell and gas boiler, and $P_{E L O A D}, P_{E L Z}, P_{H L O A D}, P_{H T}$ are power consumption of electrical load, electrolyzer, heat load and heater, respectively.

Operation Strategy. For purpose of minimizing overall cost of the multi-energy station, the following optimization problem should be considered 


$$
\min C=\sum_{k=1}^{N}\left[P_{G R I D}(k) C_{G R I D}(k)+P_{E L Z}(k) C_{E L Z}+P_{F C}(k) C_{F C}+P_{G B}(k)\left(C_{G B}+C_{G A S}\right)-\Delta n_{S A L E}(k) C_{H 2}\right]
$$

where $C_{E L Z}, C_{F C}, C_{G B}$ are operational and maintenance costs of equipment, $C_{G A S}, C_{H 2}, C_{G R I D}$ are market prices of gas, hydrogen and external electricity, and $\Delta n_{S A L E}$ is molar quantity sold from the station during current sample time. In this problem $P_{P V}, P_{E L O A D}, P_{H L O A D}, \Delta n_{S A L E}$ are disturbing variables, and $P_{G B}, U_{e}, I_{f}$ are control variables to be decided.

Besides constraints Eq. 1-10, the following limits should also be included

$$
\begin{gathered}
P_{i, \text { min }} \leq P_{i}(k) \leq P_{i, \text { max }}, i \in\{E L Z, F C, G B\}, \\
T_{i, \min } \leq T_{i}(k) \leq T_{i, \text { max }}, i \in\{e, f, h\}, \\
0 \leq \sum_{j}^{k}\left[\Delta n_{H 2}(j)-\Delta n_{S A L E}(j)\right] \leq n_{\max } .
\end{gathered}
$$

\section{Case Study}

The proposed operation model is validated with a given example of electricity-heat-hydrogen energy station as shown in Fig. 1. Parameters of various equipment, related cost prices, as well as curves of electrical load, heat load, photovoltaic generation and hydrogen demand are given in this particular case, which are not listed here due to space limit. Operation for one day (96 steps, with sample time of 15 minutes) are simulated.

Simulation results of decision variables $P_{G B}, U_{e}, I_{f}$ are presented in Fig. 4, which illustrates the optimal control of gas boiler, electrolyzer and fuel cell so as to minimize total cost and improve distribution efficiency by appropriate energy conversion.
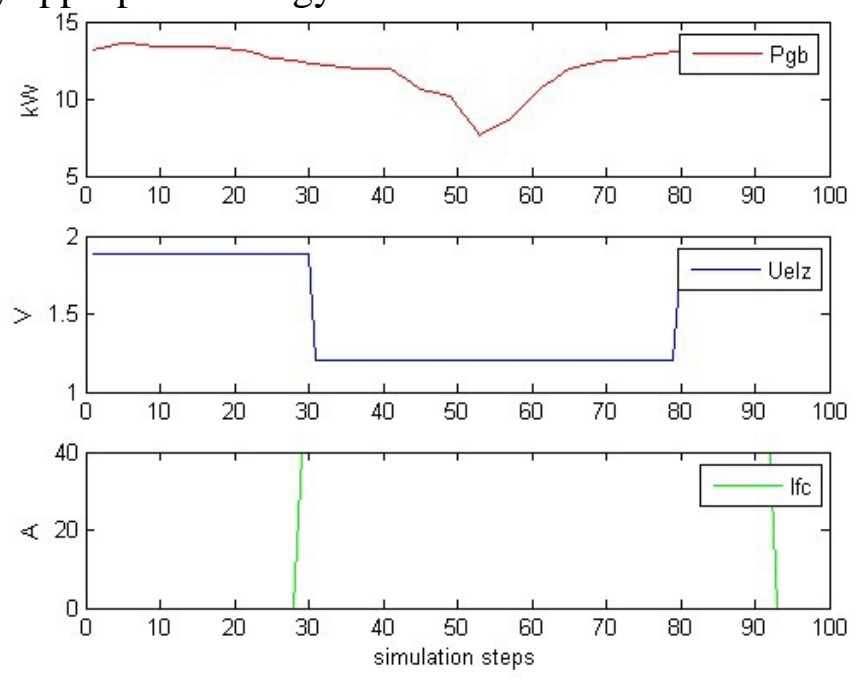

Fig. 4 Simulation results of control variables

The variation of station operating state under the optimal control of Fig. 4 is demostrated in Fig. 5. Fuel cell mainly runs during daytime while electrolyzer decreases its power consumption. It is probably because valley price of external electricity of nighttime is more ecnomical for producing enough hydrogen to meet local fuel demand. 


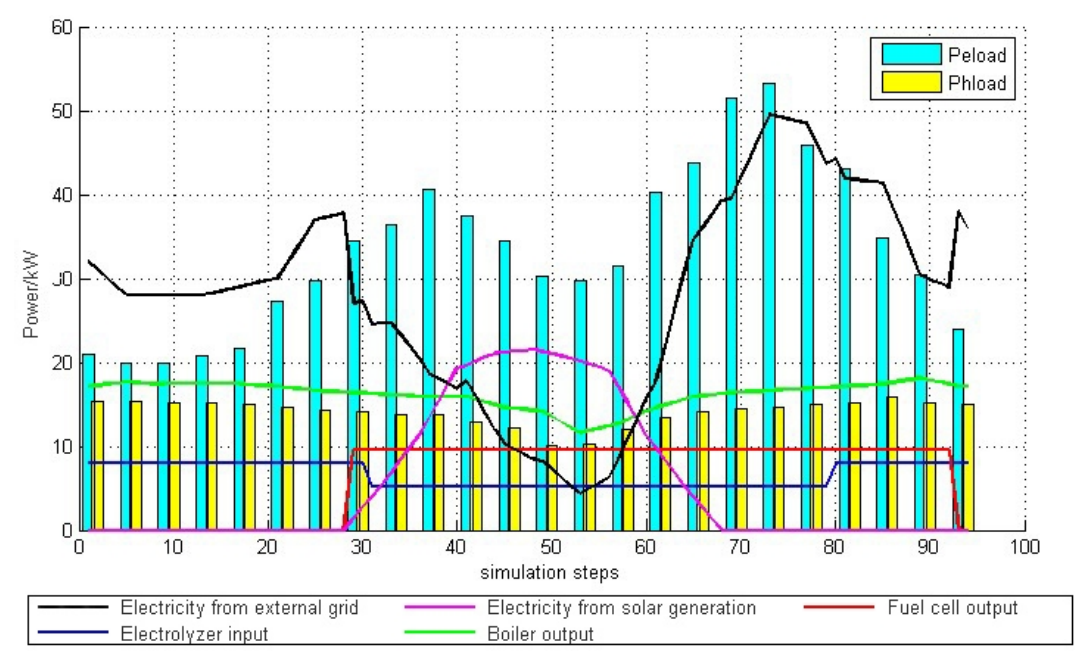

Fig. 5 Simulation results of different equipments in the station

\section{Conclusion}

An operation model and corresponding strategy of electricity-heat-hydrogen energy station is proposed in this paper. The simulation analysis proves that this multi-energy system can effectively improve the flexibility and efficiency of energy distribution.

\section{Acknowledgements}

This work was supported by Science and Technology Project of State Grid Sichuan Electric Power Company (52199716002Q).

\section{References}

[1] Dincer, I. and C. Acar, Review and evaluation of hydrogen production methods for better sustainability. International Journal of Hydrogen Energy, 2015. 40(34): p. 11094-11111.

[2] Yilanci, A., I. Dincer, and H.K. Ozturk, A review on solar-hydrogen/fuel cell hybrid energy systems for stationary applications. Progress in Energy and Combustion Science, 2009. 35(3): p. 231-244.

[3] Valverde, L., et al., Modeling, simulation and experimental set-up of a renewable hydrogen-based domestic microgrid. International Journal of Hydrogen Energy, 2013. 38(27): p. 11672-11684.

[4] Cau, G., et al., Energy management strategy based on short-term generation scheduling for a renewable microgrid using a hydrogen storage system. Energy Conversion and Management, 2014. 87: p. 820-831.

[5] Al-Baghdadi, M.A.R.S., Modelling of proton exchange membrane fuel cell performance based on semi-empirical equations. Renewable Energy, 2005. 30(10): p. 1587-1599.

[6] García-Valverde, R., N. Espinosa, and A. Urbina, Simple PEM water electrolyser model and experimental validation. International Journal of Hydrogen Energy, 2012. 37(2): p. 1927-1938.

[7] Zeng, K. and D. Zhang, Recent progress in alkaline water electrolysis for hydrogen production and applications. Progress in Energy and Combustion Science, 2010. 36(3): p. 307-326.

[8] Thavlov, A. and H.W. Bindner, Utilization of Flexible Demand in a Virtual Power Plant Set-Up. IEEE Transactions on Smart Grid, 2015. 6(2): p. 640-647. 